\title{
Argon Ion Beam Alters Grain Structure of Copper in Surface Preparation for EBSD*
}

\author{
R.H. Geiss and D.T. Read \\ Materials Reliability Division, National Institute of Standards and Technology, Boulder, CO
}

In preparing specimens of thin films of copper for examination by electron backscatter diffraction (EBSD), surface preparation is often necessary to produce acceptable EBSD patterns. Typically, removal of a rough, damaged, or oxidized layer of the surface is required. Controlled erosion of the surface with an argon ion beam is a common technique. On applying recommended etching conditions to rough surfaces of pure copper films, we observed that the grain structure appeared to change. To further investigate this, we performed a series of controlled experiments on both thin plated films and on bulk copper. We chose as a starting structure samples that were prepared by electrochemical polishing. Secondary electron (SE) images and orientation maps deduced from EBSD data were used to evaluate the grain structures. The orientation maps were inverse pole figures (IPF) constructed in the plane normal direction (ND) as well as in an in-plane orthogonal direction (RD).

IPF maps of the structure of a copper film, eight $\mu \mathrm{m}$ thick, plated on silicon, are shown in figure 1, after four minutes of electrochemical polishing. The same sample was then etched in an argon ion beam. The operating conditions, following the recommendations in [1], were: $7.5 \mathrm{kV}, 4.39$ X 10-2 $\mathrm{Pa}(3.3 \mathrm{X} 10-4$ Torr), $180 \mu \mathrm{A}, 70^{\circ}$ tilt relative to sample normal and slow rotation about the sample normal. The argon etching was done in 15 minute increments, with EBSD data collected after each 15 minute increment. IPF maps from the same area of the sample in both the ND and RD are shown in figures 2 through 4, for 15,30 and 60 minutes total etching, respectively. It is easily seen that some grains grew quite rapidly in each time increment. Grains that were large initially seemed to grow most in the first 15 minutes. After 30 minutes of argon etching and especially after 60 minutes, many of the grains that were initially small also grew quite large. In all the figures, the IPF map on the left is for the ND, and the IPF map on the right is for the RD. The dominance of the color blue in the ND IPF in the ND IPF of the starting sample demonstrates a strong $<111>$ ND orientation, which is frequently observed in plated copper films. The RD IPF shows a mixture of $<011>$ and $<112>$ orientations. The largest grains, which are the first to grow, were not oriented in $<111>$ ND as seen by the different colors. However, after 30 minutes of argon etching, some grains with $<111>$ ND orientations could be observed to grow. On the other hand, even after 60 minutes of argon etching, there were grains with initial $<111>$ ND orientations that did not grow. These differences could be caused by structural features not visible on the exposed surface. The SE images in figure 5 further demonstrate the changes in the surface structure that occurred after 60 minutes of argon etching.

To determine whether this grain growth is only observed in thin films, we performed a similar experiment on bulk copper. The results are shown in figure 6 , which shows a ND IPF before and after 30 minutes of argon etching. Although the differences are not nearly as significant, careful inspection of the two images does show grain growth.

1. Nowell, M.M., Journal of Electronic Materials 31, (1), 23-32, 2002.

* Contribution of the U.S. Department of Commerce, National Institute of Standards and Technology. Not subject to copyright in the U.S.A. 


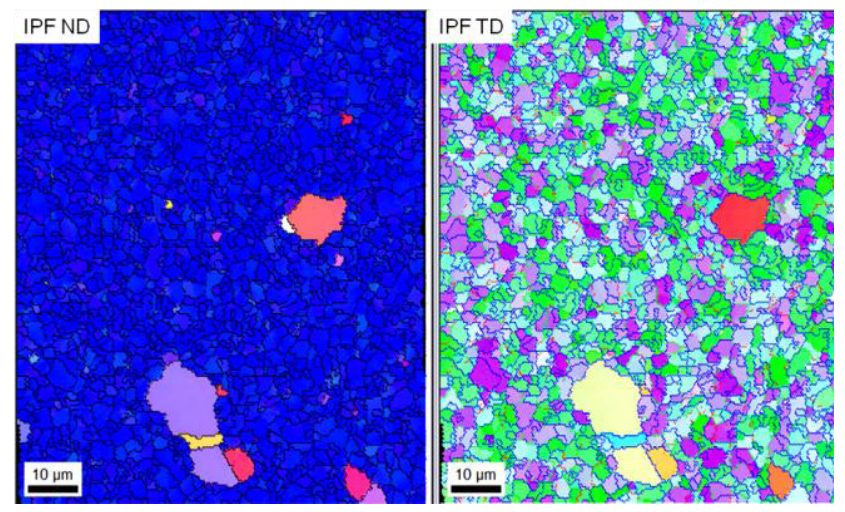

Figure 1. Plated copper film after 4 minutes of electropolishing.
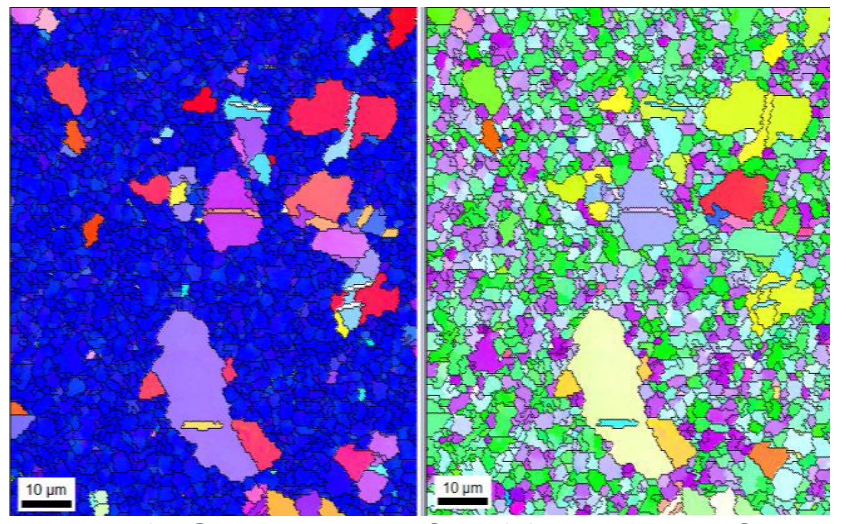

Figure 3. Same area after 30 minutes of argon etching.

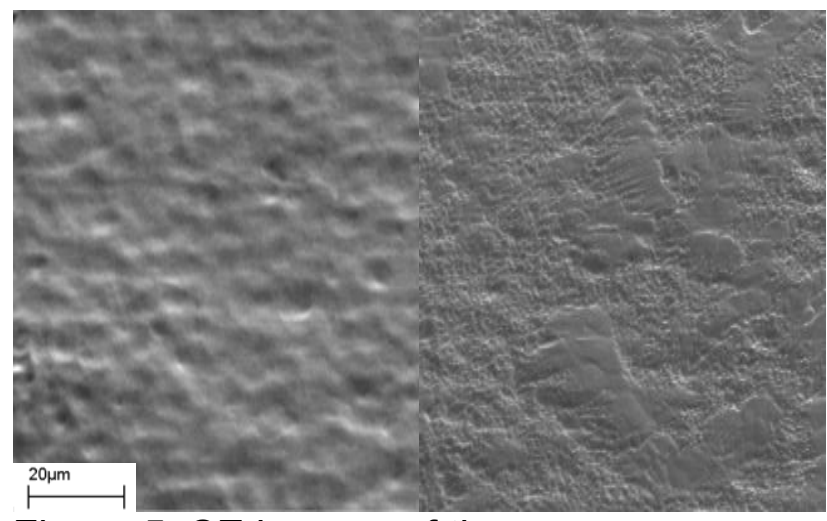

Figure 5. SE images of the same area used in the EBSD mapping. Image on left is after electropolishing. Image on right is after 60 minutes of argon etching.
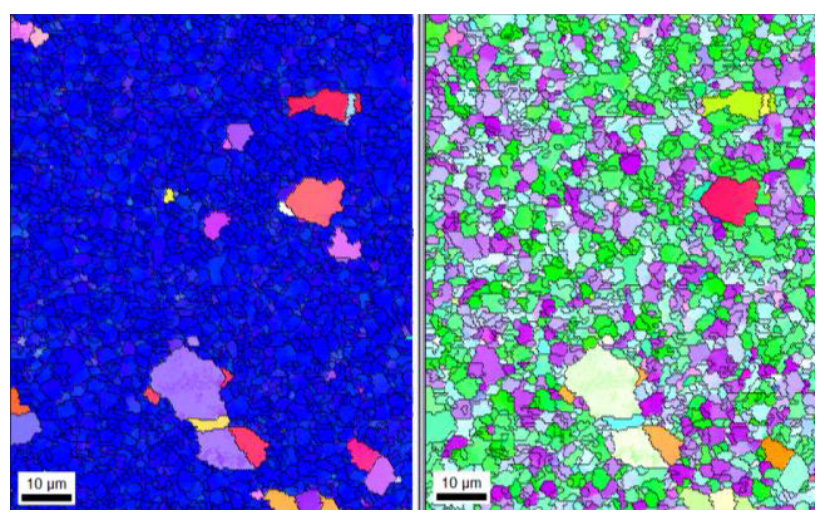

Figure 2. Same area after 15 minute of argon etching.

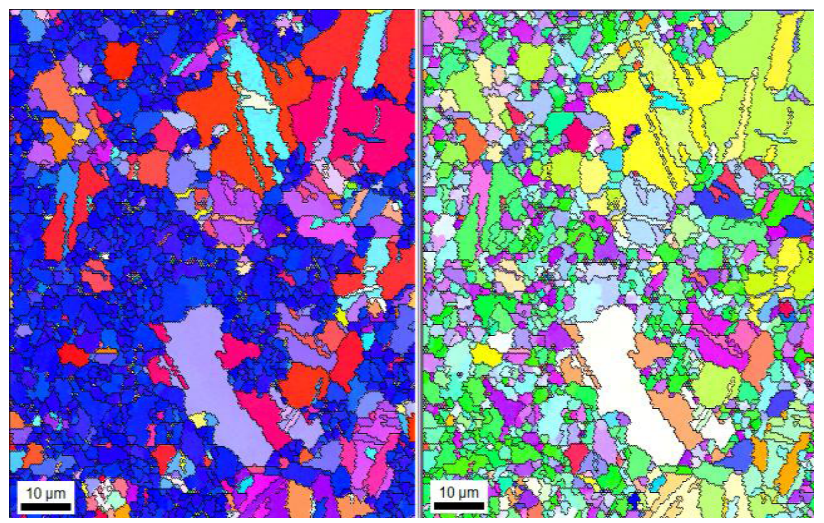

Figure 4. Same area after 60 minutes of argon etching.

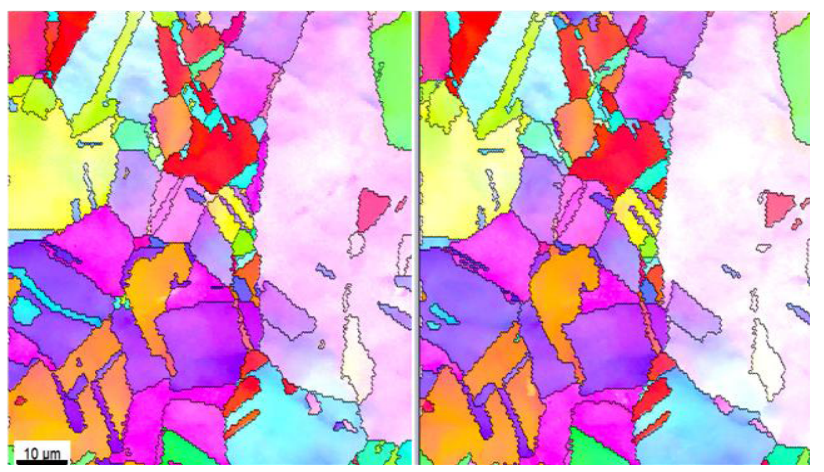

Figure 6. ND IPF maps from bulk copper sample. The image on left is after electropolishing. The image on the right is after 30 minutes of argon etching. 